TRANSACTIONS OF THE

AMERICAN MATHEMATICAL SOCIETY

Volume 364, Number 9, September 2012, Pages 4533-4549

S 0002-9947(2012)05414-6

Article electronically published on March 21, 2012

\title{
ON THE GLOBAL SOLVABILITY FOR OVERDETERMINED SYSTEMS
}

\author{
ADALBERTO P. BERGAMASCO, ALEXANDRE KIRILOV, \\ WAGNER VIEIRA LEITE NUNES, AND SÉRGIO LUÍS ZANI
}

\begin{abstract}
We consider a class of systems of two smooth vector fields on the 3 -torus associated to a closed 1-form. We prove that the global solvability is completely determined by the connectedness of the sublevel and superlevel sets of a primitive of this 1 -form in the minimal covering.
\end{abstract}

\section{INTRODUCTION}

Let $\mathbb{T}^{n}=(\mathbb{R} / 2 \pi \mathbb{Z})^{n}$ be the $n$-dimensional torus, and let $b$ be a smooth, real, closed 1-form defined on $\mathbb{T}^{2}$, and consider the line subbundle, $T^{\prime}$, of the complexified cotangent bundle $\mathbb{C} \otimes T^{*}\left(\mathbb{T}^{3}\right)$ spanned by the 1 -form $d x-i b(t) \in \bigwedge^{1} C^{\infty}\left(\mathbb{T}^{3}\right)$, where $(t, x)=\left(t_{1}, t_{2}, x\right)$ are the coordinates in $\mathbb{T}^{3}$. The orthogonal bundle $\mathcal{V}=\left(T^{\prime}\right)^{\perp}$ is then a vector subbundle of the complexified tangent bundle $\mathbb{C} \otimes T\left(\mathbb{T}^{3}\right)$ whose fibers have dimension two. The subbundle $\mathcal{V}$ is a locally integrable structure of codimension one over $\mathbb{T}^{3}$ spanned by the vector fields

$$
L_{j}=\frac{\partial}{\partial t_{j}}+i b_{j}(t) \frac{\partial}{\partial x}, \quad j=1,2 .
$$

For further information on these concepts and ideas we refer the reader to the works [18 of Treves and 13 of Berhanu, Cordaro and Hounie.

Let $d_{t}$ be the exterior derivative in $\mathbb{T}^{2}$, and consider the differential operator $\mathbb{L}^{0}$ which acts on functions - or distributions - on the torus by means of

$$
\mathbb{L}^{0} u=d_{t} u+i b(t) \wedge \frac{\partial}{\partial x} u
$$

In this work we are interested in global solvability in the sense that given any smooth $f$ satisfying natural compatibility conditions, there should exist a distribution $u$ such that $\mathbb{L}^{0} u=f$.

The gist of the present article is that the global solvability of the operator $\mathbb{L}^{0}$ is completely determined by the connectedness of the sublevel and superlevel sets of a primitive of $b$, more precisely, a primitive of the pull-back of $b$ to a convenient covering space of $\mathbb{T}^{2}$, the choice of which will depend on the periods of this 1-form.

When $b$ is an exact 1-form, there exists a global primitive of $b$ on the manifold itself and in this case the global solvability is equivalent to the connectedness of all superlevel and sublevel sets of the primitive, as has been proved by Cardoso

Received by the editors January 14, 2010 and, in revised form, July 8, 2010.

2010 Mathematics Subject Classification. Primary 35A01, 35N10, 58J10.

Key words and phrases. Global solvability, connected sublevels and superlevels, minimal covering, commensurable periods, periodic solutions.

The first and the fourth authors were partially supported by CNPq and FAPESP; the second author was partially supported by CNPq; the third author was partially supported by FAPESP.

(C)2012 American Mathematical Society 4533

Reverts to public domain 28 years from publication 
and Hounie in [14. For the proof of sufficiency the authors used an embedding of the manifold into a space of higher dimension, via Whitney's theorem, and then applied a semiglobal result from the pioneering work of Treves in [17.

When the 1 -form $b$ is closed, but nonexact, it makes sense to ask if $\mathbb{L}^{0}$ is globally solvable; on the other hand, a global primitive of $b$ does not exist on the manifold, hence it is not immediately clear what kind of condition bearing on $b$ would yield global solvability.

In order to deal with this difficulty, Bergamasco, Nunes and Zani in 8, 9, and Bergamasco and Kirilov in [6], used an approach based on the study of the properties of a primitive of the pull-back of $b$ via the universal covering $\Pi: \mathbb{R}^{2} \rightarrow \mathbb{T}^{2}$.

The results obtained in [6] concern the case when the periods of $b$ are incommensurable, that is, rationally independent. In that case, the authors proved that $\mathbb{L}^{0}$ is globally solvable if and only if the sublevels $\Omega_{r}=\left\{t \in \mathbb{R}^{2} ; B(t)<r\right\}$ and the superlevels $\Omega^{r}=\left\{t \in \mathbb{R}^{2} ; B(t)>r\right\}$ are connected, for all $r \in \mathbb{R}$, where $B(t)=\int_{t_{0}}^{t} \Pi^{*} b$ is a primitive of the pull-back of $b$ via the universal covering $\Pi: \mathbb{R}^{2} \rightarrow \mathbb{T}^{2}$.

In this work we study the remaining case, namely, the case when $b$ is closed, nonexact and has commensurable periods. Our present results provide necessary and sufficient conditions for the global solvability by means of an analysis of the connectedness of superlevel and sublevel sets of a primitive of a pull-back of $b$ to the cylinder $\mathbb{T} \times \mathbb{R}$.

In fact, we present a characterization of global solvability by means of what we call a minimal covering of $\mathbb{T}^{2}$ with respect to the 1 -form $b$, in such a way as to unify the results obtained in 6, 14, with the ones proved in the present work.

Other works dealing with global properties such as solvability and hypoellipticity of systems of vector fields are [2, 3, 12, while [1, 4, 15, 7, 10, 11, 16, treat the case of a single vector field.

This article is organized as follows. In section 2 we present more details of the problem and two equivalent formulations of our main result. In section 3 we consider a model case and present the construction of $f$ satisfying the natural compatibility conditions such that $\mathbb{L}^{0} u=f$ has no solution. In section 4 we show that the general case (with disconnected superlevels or sublevels) can be reduced to the model case by diffeomorphisms of the torus. To reach this reduction, we make use of Sard's lemma and some properties of regular level sets. In section 5 we prove that the condition of connected superlevel and sublevel sets is sufficient for global solvability.

\section{Preliminaries And Statement of the main theOrem}

In this work the property which will play a decisive role regarding the global solvability of $\mathbb{L}^{0}$ is the connectedness of sublevel and superlevel sets of a global primitive of a pull-back of $b$ to a convenient covering space of $\mathbb{T}^{2}$. The choice of such a covering will depend on the periods

$$
b_{10}=\frac{1}{2 \pi} \int_{0}^{2 \pi} b_{1}\left(\tau_{1}, 0\right) d \tau_{1} \quad \text { and } \quad b_{20}=\frac{1}{2 \pi} \int_{0}^{2 \pi} b_{2}\left(0, \tau_{2}\right) d \tau_{2} .
$$

The group of periods of $b$ is defined by

$$
\operatorname{Per}(b) \doteq\left\{\frac{1}{2 \pi} \int_{\sigma} b ; \sigma \text { is a loop in } \mathbb{T}^{2}\right\} ;
$$

equivalently, $\operatorname{Per}(b)$ is the additive subgroup of $\mathbb{R}$ consisting of all integral combinations of the numbers $b_{10}, b_{20}$. 
The possible values for $r \doteq \operatorname{rank}(\operatorname{Per}(b))$ are $r=0,1$, and 2 .

$i$. If $r=0$, the 1 -form $b$ is exact, that is, there exists $B \in C^{\infty}\left(\mathbb{T}^{2} ; \mathbb{R}\right)$ with $d B=b$.

$i i$. If $r=1$, the periods $b_{10}, b_{20}$ are rationally dependent but are not both equal to 0 ; in this case we say that the periods of $b$ are commensurable.

iii. If $r=2$, the periods $b_{10}, b_{20}$ are rationally independent; here we say that the periods of $b$ are incommensurable.

We now present the definition of a minimal covering.

Definition 2.1. Let $b$ be a smooth, real, closed 1-form defined on the two-dimensional torus $\mathbb{T}^{2}$. A minimal covering of $\mathbb{T}^{2}$, with respect to the 1 -form $b$, is a covering space $\Pi: \mathcal{T} \rightarrow \mathbb{T}^{2}$ such that $\Pi^{*} b$ is exact and furthermore such that there is no smaller covering with the same property.

Remark 2.2. We have three possibilities for minimal coverings of $\mathbb{T}^{2}$, with respect to the 1 -form $b$, namely:

$i$. if $b$ is exact, then $\mathcal{T}=\mathbb{T}^{2}$;

ii. if the periods of $b$ are commensurable, then $\mathcal{T} \simeq \mathbb{T} \times \mathbb{R}$ (diffeomorphic);

iii. if the periods of $b$ are incommensurable, then $\mathcal{T}=\mathbb{R}^{2}$.

We now present our definition of global solvability.

Definition 2.3. Let $\mathbb{E}$ be the set of all $f_{1} d t_{1}+f_{2} d t_{2} \in C^{\infty}\left(\bigwedge^{1}\left(\mathbb{T}^{3}\right)\right)$ satisfying $L_{1} f_{2}=L_{2} f_{1}$ and $\int_{\mathbb{T}^{2}} f_{j}(t, x) d t_{j} d x=0, j=1,2$.

The operator $\mathbb{L}^{0}$ is said to be globally solvable on $\mathbb{T}^{3}$ if for any $f \in \mathbb{E}$ there exists $u \in \mathcal{D}^{\prime}\left(\mathbb{T}^{3}\right)$ satisfying $\mathbb{L}^{0} u=f$.

It is not difficult to prove that if $f \doteq f_{1} d t_{1}+f_{2} d t_{2} \in C^{\infty}\left(\bigwedge^{1}\left(\mathbb{T}^{3}\right)\right)$ is such that there is $u \in \mathcal{D}^{\prime}\left(\mathbb{T}^{3}\right)$ with $\mathbb{L}^{0} u=f$, then $\int_{\mathbb{T}^{2}} f_{j}(t, x) d t_{j} d x=0, j=1,2$ and $L_{1} f_{2}=L_{2} f_{1}$. This supports our definition. We are now ready to state our main result.

Theorem 2.4. Let $b \in \bigwedge^{1} C^{\infty}\left(\mathbb{T}^{2}\right)$ be a closed 1 -form and set $B(t) \doteq \int_{0}^{t} \Pi^{*} b$, where $\Pi: \mathcal{T} \rightarrow \mathbb{T}^{2}$ is the minimal covering, in the sense of Definition 2.1 , and $\Pi^{*} b$ is the pull-back of $b$. The operator $\mathbb{L}^{0}=d_{t}+i b(t) \wedge \partial / \partial x$ is globally solvable if and only if the sublevels $\Omega_{r}=\{\tau \in \mathcal{T} ; B(\tau)<r\}$ and the superlevels $\Omega^{r}=\{\tau \in \mathcal{T} ; B(\tau)>r\}$ are connected, for every $r \in \mathbb{R}$.

Remark 2.5. In order to prove the theorem above we will make use of diffeomorphisms of $\mathbb{T}^{2}$ and also of the induced diffeomorphisms of the covering space. Note that if $\mathcal{T}$ and $\mathcal{T}^{\prime}$ are covering spaces and $\Phi: \mathcal{T}^{\prime} \rightarrow \mathcal{T}$ is a diffeomorphism, then the connectedness of all superlevels (sublevels) of a continuous function $B: \mathcal{T} \rightarrow \mathbb{R}$ is equivalent to the connectedness of all superlevels (sublevels) of $B \circ \Phi$.

We now make some remarks and provide examples with the purpose of clarifying the assumptions of connectedness in the theorem.

If $b$ is an exact 1 -form it is essential to consider the minimal covering in the above theorem as the following example shows: if $L_{1}=\partial / \partial t_{1}-i \sin t_{1} \partial / \partial x, L_{2}=\partial / \partial t_{2}$, then $B\left(t_{1}, t_{2}\right)=\cos t_{1}$ has connected sublevel and superlevel sets both in $\mathbb{T}^{2}$ (hence $\mathbb{L}^{0}$ is globally solvable) and in $\mathbb{T}_{t_{1}} \times \mathbb{R}_{t_{2}}$, but neither in $\mathbb{R}^{2}$ nor in $\mathbb{R}_{t_{1}} \times \mathbb{T}_{t_{2}}$.

If $b$ is a closed nonexact 1 -form with commensurable periods, then up to a diffeomorphism of the torus $\mathbb{T}^{2}$, the minimal covering space is $\mathcal{T}=\mathbb{T} \times \mathbb{R}$. Observe that, if we set $B_{1}(t) \doteq \int_{0}^{t} \Pi_{1}^{*} b$ and $B_{2}(t) \doteq \int_{0}^{t} \Pi_{2}^{*} b$, where $\Pi_{1}: \mathbb{T} \times \mathbb{R} \rightarrow \mathbb{T}^{2}$ is the 
minimal covering and $\Pi_{2}: \mathbb{R}^{2} \rightarrow \mathbb{T}^{2}$ is the universal covering, then for each $r \in \mathbb{R}$ we have:

i. $\Omega_{1, r}$ is connected if and only if $\Omega_{2, r}$ is connected,

ii. $\Omega_{1}^{r}$ is connected if and only if $\Omega_{2}^{r}$ is connected, where $\Omega_{j, r} \doteq\left\{\tau \in \mathbb{R}^{2} ; B_{j}(\tau)<r\right\}$ and $\Omega_{j}^{r} \doteq\left\{\tau \in \mathbb{T} \times \mathbb{R} ; B_{j}(\tau)>r\right\}$ for $j=1,2$.

On the other hand, when $\Omega_{1}^{r}$ and $\Omega_{2}^{r}$ are disconnected, the number of connected components of these two sets is, in general, different. For example, if $b\left(t_{1}, t_{2}\right)=$ $\cos \left(t_{1}+t_{2}\right) d t_{1}+\left(\cos \left(t_{1}+t_{2}\right)-1 / 2 \pi\right) d t_{2}$, then $B\left(t_{1}, t_{2}\right)=\sin \left(t_{1}+t_{2}\right)-t_{2} / 2 \pi$, with $\left(t_{1}, t_{2}\right) \in \mathbb{T} \times \mathbb{R}$. Taking $r=0$ we have that $\Omega_{1}^{0}$ has only two connected components, while $\Omega_{2}^{0}$ has infinitely many connected components. The same result is true for $\Omega_{1,0}$ and $\Omega_{2,0}$.

In view of these remarks, we may rewrite Theorem 2.4 as follows.

Theorem 2.6. Let $b$ be a smooth closed 1 -form on $\mathbb{T}^{2}$ and $\mathbb{L}^{0}=d_{t}+i b(t) \wedge \partial / \partial x$.

i. If $b$ is exact and $B(t) \doteq \int_{0}^{t} b$, then the operator $\mathbb{L}^{0}$ is globally solvable if and only if the sublevels $\left\{\tau \in \mathbb{T}^{2} ; B(\tau)<r\right\}$ and the superlevels $\left\{\tau \in \mathbb{T}^{2} ; B(\tau)>\right.$ $r\}$ are connected, for every $r \in \mathbb{R}$.

ii. If $b$ is nonexact and $B(t) \doteq \int_{0}^{t} \Pi^{*} b$, where $\Pi: \mathbb{R}^{2} \rightarrow \mathbb{T}^{2}$ is the universal covering, then the operator $\mathbb{L}^{0}$ is globally solvable if and only if the sublevels $\left\{\tau \in \mathbb{R}^{2} ; B(\tau)<r\right\}$ and the superlevels $\left\{\tau \in \mathbb{R}^{2} ; B(\tau)>r\right\}$ are connected, for every $r \in \mathbb{R}$.

Taking into account the results of [6] and [14, we need only analyze the commensurable case.

\section{NeCessity: The MOdel CASe}

In this section we show that, in a special situation, which we will refer to as the model case, we are able to construct an $f \in \mathbb{E}$ (see Definition 2.3) for which the equation $\mathbb{L}^{0} u=f$ has no solution $u \in \mathcal{D}^{\prime}\left(\mathbb{T}^{3}\right)$.

We will call the model case the case when the 1-form $b$ has the following properties: $b_{20}<b_{10}=0,(0,0) \in \Pi^{-1}(\Sigma)$, where $\Sigma \doteq\left\{t \in \mathbb{T}^{2}: b(t)=0\right\}$ is the critical set of $b, B(0,0)=0$ and $M>M^{\prime} \geqslant 0$, where

$$
M \doteq \max _{t \in[0,2 \pi]^{2}} B(t)=B\left(0, t_{2}^{*}\right) \text { and } M^{\prime} \doteq \max _{t_{1} \in[0,2 \pi]} B\left(t_{1}, 0\right) .
$$

Let us consider $M_{1}$ and $\delta>0$ such that $M^{\prime}<M_{1}<M$ and

$$
\max \left\{B\left(t_{1}, t_{2}\right)-B\left(0, t_{2}\right): 0 \leqslant t_{1} \leqslant 2 \pi,\left|t_{2}\right| \leqslant \delta\right\} \leqslant M_{1} .
$$

In order to exhibit an $f=f_{1} d t_{1}+f_{2} d t_{2} \in \mathbb{E}$ such that the equation $\mathbb{L}^{0} u=f$, or equivalently, $L_{1} u=f_{1}, L_{2} u=f_{2}$, has no solution $u \in \mathcal{D}^{\prime}\left(\mathbb{T}^{3}\right)$, we need to verify that the compatibility condition $\mathbb{L}^{1} f=0$ or, equivalently, $L_{1} f_{2}=L_{2} f_{1}$ is satisfied.

We choose $f_{1} \equiv 0$; then $f_{2}$ must verify $L_{1} f_{2}=0$. We expand $f_{2}$ in its $x$-Fourier series

$$
f_{2}(t, x)=\frac{1}{2 \pi} \sum_{n=-\infty}^{\infty} \hat{f}_{2}(t, n) e^{i n x} .
$$

Any possible solution to the equation $L_{2} u=f_{2}$ can be written in the form of an $x$-Fourier series, namely,

$$
u(t, x)=\frac{1}{2 \pi} \sum_{n=-\infty}^{\infty} \hat{u}(t, n) e^{i n x} .
$$


In fact, the partial Fourier coefficients of $u$ must be solutions to

$$
\left(\partial / \partial t_{2}-n b_{2}\left(t_{1}, t_{2}\right)\right) \hat{u}(t, n)=\hat{f}_{2}(t, n), \quad n \in \mathbb{Z} .
$$

Since $b_{20}<0$, we have $1-e^{2 \pi n b_{20}} \neq 0$, for all $n \neq 0$. It follows that when $n \neq 0$ this equation has a unique solution given by

$$
\hat{u}(t, n)=d_{2 n} \int_{0}^{2 \pi} e^{-i n\left[B\left(t_{1}, t_{2}\right)-B\left(t_{1}, t_{2}-s_{2}\right)\right]} \hat{f}_{2}\left(t_{1}, t_{2}-s_{2}, n\right) d s_{2},
$$

where $d_{2 n} \doteq\left(1-e^{2 \pi n b_{20}}\right)^{-1}$.

In fact, we will use only positive frequencies and, thus, look for $f_{2}$ of the form

$$
f_{2}(t, x)=\frac{1}{2 \pi} \sum_{n=1}^{\infty} \hat{f}_{2}(t, n) e^{i n x}
$$

then, up to a constant, any possible solution to $L_{2} u=f_{2}$ can be written as

$$
u(t, x)=\frac{1}{2 \pi} \sum_{n=1}^{\infty} \hat{u}(t, n) e^{i n x}
$$

Now we proceed to make the choice of each $\hat{f}_{2}(t, n)$, with $n \geqslant 1$. Let us consider an auxiliary function $\chi_{\delta} \in C_{c}^{\infty}(\mathbb{R})$ satisfying the conditions

$$
\begin{aligned}
& \chi_{\delta}\left(t_{2}\right)=1, \text { if }\left|t_{2}\right|<\delta, \\
& \chi_{\delta}\left(t_{2}\right)=0, \text { if }\left|t_{2}\right| \geqslant 2 \delta, \\
& 0 \leqslant \chi_{\delta}\left(t_{2}\right) \leqslant 1, t_{2} \in \mathbb{R},
\end{aligned}
$$

where $\delta>0$ is as in (3.2). If necessary, we take a smaller $\delta$ so that $0<2 \delta<t_{2}^{*}<$ $2 \pi-2 \delta$, where $t_{2}^{*}$ is as in (3.1), and we consider the smooth $2 \pi$-periodic function $q: \mathbb{R}^{2} \rightarrow \mathbb{R}$ such that, for $\left(t_{1}, t_{2}\right) \in Q^{*} \doteq[0,2 \pi] \times[-\pi, \pi]$ it is given by

$$
q\left(t_{1}, t_{2}\right)=q\left(t_{2}\right) \doteq\left(t_{2}^{2}-1\right) \cdot \chi_{\delta}\left(t_{2}\right)+1 \text {. }
$$

Finally, for each $\left(t_{1}, t_{2}\right) \in \mathbb{R}^{2}$, we define

$$
\hat{f}_{2}\left(t_{1}, t_{2}, n\right) \doteq e^{n\left[\varepsilon+B\left(t_{1}, t_{2}\right)-B\left(0, t_{2}\right)-M-K q\left(t_{2}\right)\right]}, n \geqslant 1,
$$

where $\varepsilon>0$ and $K>0$ will be chosen later on.

Proposition 3.1. There are $\varepsilon>0$ and $K>0$ such that if $f_{1}=0$ and $f_{2}$ is as above, then $f \doteq f_{1} d t_{1}+f_{2} d t_{2} \in \mathbb{E}$. Furthermore, the same conclusion will hold if we replace $\varepsilon>0$ by any $\varepsilon^{\prime}>0$ with $0<\varepsilon^{\prime}<\varepsilon$, and also if we replace $K>0$ by any $K^{\prime}>0$ with $0<K<K^{\prime}$.

Proof. We will prove that $f_{2} \in C^{\infty}\left(\mathbb{T}^{3}\right)$ and $L_{1} f_{2}=0$.

It is easy to see that, for each $n \in \mathbb{N}, \hat{f}_{2}(\cdot, \cdot, n)$ is $2 \pi$-periodic in both variables (recall that $\left.b_{10}=0\right)$ and $\left(\partial / \partial t_{1}-n b_{1}\left(t_{1}, t_{2}\right)\right) \hat{f}_{2}\left(t_{1}, t_{2}, n\right)=0$, for all $\left(t_{1}, t_{2}\right) \in \mathbb{R}^{2}$; as soon as we prove that $f_{2}$ is smooth, it will follow from this that $L_{1} f_{2}=0$. Since $f_{1}=0$, we will have $L_{1} f_{2}=L_{2} f_{1}$.

Recall that, by (3.2), $B\left(t_{1}, t_{2}\right)-B\left(0, t_{2}\right) \leqslant M_{1}$ for all $0 \leqslant t_{2}<\delta$. Since $q(t) \geqslant 0$ for all $t \in[0,2 \pi]^{2}$, if we choose $0<\varepsilon<M-M_{1}$, we have

$$
\varepsilon+B\left(t_{1}, t_{2}\right)-B\left(0, t_{2}\right)-M-K q\left(t_{2}\right) \leqslant \varepsilon+M_{1}-M<0 .
$$

Note that, if $0<2 \pi-t_{2}<\delta$, then

$$
B\left(t_{1}, t_{2}\right)-B\left(0, t_{2}\right)=B\left(t_{1}, t_{2}-2 \pi\right)-B\left(0, t_{2}-2 \pi\right)<M_{1} .
$$

Thus, we also have (3.7) for $0<2 \pi-t_{2}<\delta$. 
If $t \in[0,2 \pi]^{2}$ and $\delta \leqslant t_{2} \leqslant \pi$, then $q\left(t_{2}\right) \geqslant\left(\delta^{2}-1\right) \chi_{\delta}\left(t_{2}\right)+1 \geqslant \delta^{2}$, since $\chi_{\delta}\left(t_{2}\right) \leq$ 1. If $t \in[0,2 \pi]^{2}$ and $\pi \leqslant t_{2} \leqslant 2 \pi-\delta$, then $q\left(t_{2}\right)=\left(\left(t_{2}-2 \pi\right)^{2}-1\right) \chi_{\delta}\left(t_{2}-2 \pi\right)+1 \geqslant \delta^{2}$. Now choosing $K$ such that

$$
K>\frac{\varepsilon-m}{\delta^{2}}
$$

where $m \doteq \min \left\{B\left(0, t_{2}\right) ; 0 \leqslant t_{2} \leqslant 2 \pi\right\}$, we have

$$
\varepsilon+B\left(t_{1}, t_{2}\right)-B\left(0, t_{2}\right)-M-K q\left(t_{2}\right)<\varepsilon-m-K \delta^{2}<0,
$$

for all $t \in[0,2 \pi]^{2}$ with $\delta \leqslant t_{2} \leqslant 2 \pi-\delta$.

Finally, if $\lambda \doteq \max \left\{\varepsilon+M_{1}-M, \varepsilon-m-K \delta^{2}\right\}$, we have $\lambda<0$ and

$$
\left|\hat{f}_{2}(t, n)\right|=e^{n\left[\varepsilon+B\left(t_{1}, t_{2}\right)-B\left(0, t_{2}\right)-M-K q\left(t_{2}\right)\right]} \leqslant e^{n \lambda},
$$

for all $t \in[0,2 \pi]^{2}$ and $n \in \mathbb{N}$.

Now, for $\alpha, \beta \in \mathbb{Z}_{+}$, we may write $\partial^{(\alpha, \beta)} \hat{f}_{2}(t, n)=\hat{f}_{2}(t, n) P(t, n, \alpha, \beta)$, where $P(t, n, \alpha, \beta)$ satisfies

$$
|P(t, n, \alpha, \beta)| \leqslant C_{\alpha, \beta} n^{\alpha+\beta}, \forall n \in \mathbb{N} \text { and } \forall t \in[0,2 \pi]^{2},
$$

for some $C_{\alpha, \beta}>0$.

Therefore

$$
\left|\partial^{(\alpha, \beta)} \hat{f}_{2}(t, n)\right|=\left|\hat{f}_{2}(t, n)\right||P(t, n, \alpha, \beta)| \leqslant C_{\alpha, \beta} n^{\alpha+\beta} e^{n \lambda},
$$

for all $n \in \mathbb{N}$ and $t \in[0,2 \pi]^{2}$, hence $f_{2} \in C^{\infty}\left(\mathbb{T}^{3}\right)$.

In view of the fact that $f_{2}$ contains only nonzero frequencies, it is easy to see that we have $\int_{\mathbb{T}^{2}} f_{2}(t, x) d t_{2} d x=0$.

Since we trivially have $\int_{\mathbb{T}^{2}} f_{1}(t, x) d t_{1} d x=0$, the proof is complete.

We now proceed to state the main result of this section.

Proposition 3.2. There exists no periodic distribution whose sequence of $x$-Fourier coefficients is given by (3.4) and (3.6).

Proof. We will analyze the behavior of $\hat{u}\left(0, t_{2}^{*}, n\right)$, where $t_{2}^{*}$ is as in (3.1). From (3.4) and (3.6) we have

$$
\hat{u}(t, n)=d_{2 n} \int_{0}^{2 \pi} e^{n\left[\phi\left(t_{1}, t_{2}, s_{2}\right)+\varepsilon\right]} d s_{2}
$$

where

$$
\phi\left(t_{1}, t_{2}, s_{2}\right)=\left[B\left(t_{1}, t_{2}\right)-B\left(0, t_{2}-s_{2}\right)-M-K q\left(t_{2}-s_{2}\right)\right] .
$$

We observe that $\phi\left(0, t_{2}^{*}, s_{2}\right)=-B\left(0, t_{2}^{*}-s_{2}\right)-K q\left(t_{2}^{*}-s_{2}\right)$. From (3.9) we obtain

$$
\hat{u}\left(0, t_{2}^{*}, n\right)=d_{2 n}\left(I_{n}+J_{n}\right),
$$

where

$$
I_{n} \doteq \int_{\left|t_{2}^{*}-s_{2}\right|<\delta} e^{n\left[\phi\left(0, t_{2}^{*}, s_{2}\right)+\varepsilon\right]} d s_{2} \text { and } J_{n} \doteq \int_{\left|t_{2}^{*}-s_{2}\right|>\delta} e^{n\left[\phi\left(0, t_{2}^{*}, s_{2}\right)+\varepsilon\right]} d s_{2} .
$$

In the region $\left|t_{2}^{*}-s_{2}\right|<\delta$, we have $\chi_{\delta}\left(t_{2}^{*}-s_{2}\right)=1$, hence

$$
\phi\left(0, t_{2}^{*}, s_{2}\right)=-B\left(0, t_{2}^{*}-s_{2}\right)-K\left(t_{2}^{*}-s_{2}\right)^{2}
$$

and

$$
I_{n}=e^{n \varepsilon} \int_{\left|t_{2}^{*}-s_{2}\right|<\delta} e^{-n\left[B\left(0, t_{2}^{*}-s_{2}\right)+K\left(t_{2}^{*}-s_{2}\right)^{2}\right]} d s_{2} .
$$


We perform the change of variables $\sigma=t_{2}^{*}-s_{2}$ in (3.11) to obtain

$$
I_{n}=e^{n \varepsilon} \int_{|\sigma|<\delta} e^{-n g(\sigma)} d \sigma
$$

where $g(\sigma)=B(0, \sigma)+K \sigma^{2}$.

Since $g(0)=g^{\prime}(0)=0$ and $g^{\prime \prime}(0)=\partial b_{2} / \partial t_{2}(0,0)+2 K$, we may choose a larger $K>0$ and a smaller $\delta>0$ to obtain $g^{\prime \prime}(0)>0$ and therefore the origin will be the only critical point of the function $g$ on $|\sigma|<\delta$, which implies that there exist constants $C_{1}, C_{2}>0$ such that

$$
C_{2} \sigma^{2} \leqslant g(\sigma) \leqslant C_{1} \sigma^{2}, \forall \sigma \in(-\delta, \delta) .
$$

It follows that

$$
e^{n \varepsilon} \int_{|\sigma|<\delta} e^{-n C_{1} \sigma^{2}} d \sigma \leqslant I_{n} \leqslant e^{n \varepsilon} \int_{|\sigma|<\delta} e^{-n C_{2} \sigma^{2}} d \sigma
$$

We use new variables $\tau=\sigma \sqrt{n C_{1}}$ in the integral on the left and $\tau=\sigma \sqrt{n C_{2}}$ in the integral on the right above to obtain

$$
R_{1}(n) e^{n \varepsilon} \frac{1}{n C_{1}} \leqslant I_{n} \leqslant R_{2}(n) e^{n \varepsilon} \frac{1}{n C_{2}},
$$

where $R_{j}(n)=\int_{|\tau|<\delta \sqrt{n C_{j}}} e^{-\tau^{2}} d \tau$, for $j=1,2$.

Observe that $\left\{R_{j}(n)\right\}_{n \in \mathbb{N}}$ are increasing sequences, $j=1,2$, and

$$
R_{j}(1) \leqslant R_{j}(n) \leqslant \int_{\mathbb{R}} e^{-\sigma^{2}} d \sigma=\sqrt{\pi}, j=1,2,
$$

hence $R_{1}(1) e^{n \varepsilon} \frac{1}{n C_{1}} \leqslant I_{n} \leqslant \sqrt{\pi} e^{n \varepsilon} \frac{1}{n C_{2}}$.

Take $n_{0} \in \mathbb{N}$ such that $R_{1}(1)\left(n C_{1}\right)^{-1} \exp (n \varepsilon / 2) \geqslant 1$, for all $n \geqslant n_{0}$, which implies $I_{n} \geqslant e^{n \frac{\varepsilon}{2}}$, for all $n \geqslant n_{0}$. Since $J_{n} \geqslant 0$, it follows that

$$
\hat{u}\left(0, t_{2}^{*}, n\right)=d_{2 n}\left(I_{n}+J_{n}\right) \geqslant d_{2 n} I_{n} \geqslant d_{2 n} e^{n \frac{\varepsilon}{2}},
$$

for all $n \geqslant n_{0}$.

We have $d_{2 n} \geqslant 1 / 2$, thus $\left|\hat{u}\left(0, t_{2}^{*}, n\right)\right| \geqslant \frac{1}{2} e^{n \varepsilon / 2}$ for all $n \geqslant n_{0}$. We conclude that the sequence $\left\{\hat{u}\left(0, t_{2}^{*}, n\right)\right\}$ does not correspond to any periodic distribution.

Putting together the results of this section, we reach the conclusion that, in the model case, the operator $\mathbb{L}^{0}$ is not globally solvable in $\mathbb{T}^{3}$.

\section{Necessity: The Reduction to the model CASE}

The goal of this section is, starting from the assumption of the existence of a disconnected sublevel or superlevel set of $B(t)=\int_{0}^{t} \Pi^{*} b$, to find a smooth diffeomorphism of $\mathbb{T}^{2}$ that reduces our problem to the model case.

Since $b_{10}$ and $b_{20}$ are commensurable, by using diffeomorphisms of the twodimensional torus given by elements of $U(2, \mathbb{Z})$ (see [9, page 56]) we may assume that

$$
b_{20}<b_{10}=0 .
$$


In fact, since $b_{10}$ and $b_{20}$ are rationally dependent, there exist relatively prime integers $p$ and $q$ such that $p b_{10}+q b_{20}=0$. Take $r, s \in \mathbb{Z}$ such that $p r+q s=1$ and consider the diffeomorphism $\Psi: \mathbb{T}^{2} \rightarrow \mathbb{T}^{2}$ given by

$$
\Psi\left(t_{1}^{\prime}, t_{2}^{\prime}\right) \doteq\left(p t_{1}^{\prime}-s t_{2}^{\prime}, q t_{1}^{\prime}+r t_{2}^{\prime}\right) \doteq\left(t_{1}, t_{2}\right) .
$$

Let $B\left(t_{1}, t_{2}\right)$ be a primitive of $b$ defined on a cylinder of coordinates $\left(t_{1}, t_{2}\right)$. Thus, $B\left(t_{1}, t_{2}\right)=P\left(t_{1}, t_{2}\right)+b_{10} t_{1}+b_{20} t_{2}$, where $P$ is periodic. Then $B^{\prime} \doteq B \circ \Psi$ is also a primitive of $b$ on a cylinder of coordinates $\left(t_{1}^{\prime}, t_{2}^{\prime}\right)$. We have $B^{\prime}\left(t_{1}^{\prime}, t_{2}^{\prime}\right)=$ $P\left(p t_{1}^{\prime}-s t_{2}^{\prime}, q t_{1}^{\prime}+r t_{2}^{\prime}\right)+\left(b_{20} r-b_{10} s\right) t_{2}^{\prime}$. Hence, in these new coordinates $b$ has one null period.

Assume $b_{10}=0$. If $b_{20}<0$ we are done. If $b_{20}>0$ consider the diffeomorphism $\Psi\left(t_{1}^{\prime}, t_{2}^{\prime}\right)=\left(t_{1}^{\prime},-t_{2}^{\prime}\right) \doteq\left(t_{1}, t_{2}\right)$. Then for $B^{\prime} \doteq B \circ \Psi$ we have $b_{20}^{\prime}<0$. Thus, if $B\left(t_{1}, t_{2}\right)=P\left(t_{1}, t_{2}\right)+b_{20} t_{2}$, then $B^{\prime}\left(t_{1}^{\prime}, t_{2}^{\prime}\right) \doteq B\left(\Psi\left(t_{1}^{\prime}, t_{2}^{\prime}\right)\right)=P\left(t_{1}^{\prime},-t_{2}^{\prime}\right)-b_{20} t_{2}^{\prime}$. Hence, in the new coordinates, $b$ has one null period and a negative one.

Finally, since we are assuming that $B$ has a disconnected sublevel or superlevel set, by Remark 2.5 the same is true for $B^{\prime}$.

In order to keep the notation light we will use the same symbols for the coefficients of the 1 -form $b$ as for the coefficients of the pull-back of $b \in \Lambda^{1} C^{\infty}\left(\mathbb{T}^{2}\right)$ via the minimal covering $\Pi: \mathbb{T} \times \mathbb{R} \rightarrow \mathbb{T}^{2}$; in other words we will write

$$
\left(\Pi^{*} b\right)(t)=b_{1}(t) d t_{1}+b_{2}(t) d t_{2},
$$

where the functions $b_{1}, b_{2} \in C^{\infty}(\mathbb{T} \times \mathbb{R})$ are $2 \pi$-periodic in the second variable.

Since $\Pi^{*} b$ is a closed 1 -form, and since $b_{10}=0$, the function $B: \mathbb{T} \times \mathbb{R} \rightarrow \mathbb{R}$ given by

$$
B(t)=\int_{0}^{t} \Pi^{*} b, \quad t \in \mathbb{T} \times \mathbb{R}
$$

is a well-defined primitive of $\Pi^{*} b$.

Remark 4.1. Since $b_{10}=0$ we have $B(t)=P(t)+b_{20} \cdot t_{2}$, for all $t=\left(t_{1}, t_{2}\right) \in \mathbb{T} \times \mathbb{R}$, where $P \in C^{\infty}(\mathbb{T} \times \mathbb{R})$ is a $2 \pi$-periodic function in the second variable.

Let us denote by $\mathcal{F}(t)$ the level set of $B$ which contains $t \in \mathbb{T} \times \mathbb{R}$ and by $\mathcal{F}^{\prime}(t)$ the connected component of $\mathcal{F}(t)$ which contains $t \in \mathbb{T} \times \mathbb{R}$.

It follows from the above remark that each level set $\mathcal{F}(t)$ of $B$ is contained in a cylindric strip $\mathbb{T} \times[\alpha, \beta]$. Moreover every superlevel set and every sublevel set contain a half-cylinder $\mathbb{T} \times(-\infty, \beta)$, or $\mathbb{T} \times(\alpha, \infty)$, respectively. It is clear that each superlevel and each sublevel contains a unique unbounded connected component.

4.1. Characterization of the regular level sets of $B$. We say that the real number $r$ is a regular value of the function $B$ when there are no critical points of $B$ at level $r$; that is, if $B(t)=r$, then $d B(t) \neq 0$. When $r$ is a regular value we say that the corresponding level set is a regular level.

Definition 4.2. We say that a subset $G \subset \mathbb{T} \times \mathbb{R}$ is trivial if there exists a simply connected open subset of $\mathbb{T} \times \mathbb{R}$ containing $G$. Otherwise, we say that $G$ is nontrivial.

The following result gives a characterization of the regular levels. 
Proposition 4.3. If $B(t)$ is a regular value of $B$ and $\mathcal{F}(t)$ is the corresponding regular level set then:

$i$. Each connected component of $\mathcal{F}(t)$ is diffeomorphic to $\mathbb{T}$.

ii. Each connected component, say $\mathcal{F}^{\prime}$, of $\mathcal{F}(t)$ is contained in an open set, $V$, diffeomorphic to an annulus, which is foliated by connected components of pairwise distinct level sets, each one of these being diffeomorphic to $\mathbb{T}$. Furthermore $d B(t) \neq 0$ on this open set. Moreover, the points on one side of $\mathcal{F}^{\prime}$ belong to a sublevel set of $B$ whereas the points on the other side of $\mathcal{F}^{\prime}$ belong to a superlevel set. In particular, the level $B(t)$ occurs in $V$ only at $\mathcal{F}^{\prime}$.

iii. $\mathcal{F}(t)$ has only a finite number of connected components.

$i v$. $\mathcal{F}(t)$ has at least one nontrivial connected component.

Proof. Since $B(t)$ is a regular value, $\mathcal{F}(t)$ is an embedded one-dimensional submanifold of $\mathbb{T} \times \mathbb{R}$. It follows that each connected component of $\mathcal{F}(t)$ is diffeomorphic either to $\mathbb{T}$ or to $\mathbb{R}$. On the other hand, $\mathcal{F}(t)$ is contained in a cylindric strip $\mathbb{T} \times[c, d]$, hence the case of a component diffeomorphic to $\mathbb{R}$ cannot occur, which proves $i$.

By assumption, we have $d B(t) \neq 0$ at each point of the connected component $\mathcal{F}^{\prime}$, hence there is a local foliation by level sets of $B$. By compactness, we obtain a foliated neighborhood of $\mathcal{F}^{\prime}$ by connected components of level sets of $B$ with pairwise distinct levels. From this it is clear that all statements in $i i$ hold true.

It follows from $i i$ that each connected component of $\mathcal{F}(t)$ is isolated from the others. Recalling that the whole level set $\mathcal{F}(t)$ is contained in a compact strip $\mathbb{T} \times[c, d]$, we conclude that the number of components is finite, which proves iii.

To prove $i v$, let us suppose otherwise, that is, there exists a regular level set $\mathcal{F}\left(t_{0}\right)$ which has only trivial connected components.

In view of $i i i$, the number of regular connected components having the same level is finite, hence we may write

$$
\mathbb{T} \times \mathbb{R} \backslash \mathcal{F}\left(t_{0}\right)=\mathcal{U}_{0} \cup \bigcup_{j=1}^{m} \mathcal{U}_{j},
$$

where $\mathcal{U}_{0}$ is the unique unbounded connected component and each $\mathcal{U}_{j}$ is a bounded open set which satisfies $\partial \mathcal{U}_{j} \subseteq \mathcal{F}\left(t_{0}\right), j=1, \ldots, m$.

The function $B$ is continuous on the connected set $\mathcal{U}_{0}$ and never attains the value $B\left(t_{0}\right)$ there, hence we must have either $B(\tau)<B\left(t_{0}\right)$, for all $\tau \in \mathcal{U}_{0}$, or else $B(\tau)>B\left(t_{0}\right)$, for all $\tau \in \mathcal{U}_{0}$, which contradicts the fact that both the sublevel $\Omega_{B\left(t_{0}\right)}$ and the superlevel $\Omega^{B\left(t_{0}\right)}$ contain a half-cylinder.

We remark that Proposition $4.3 i i$ is valid assuming only that $\mathcal{F}^{\prime}$ is a connected component of a level set such that $d B(p) \neq 0$ for each $p \in \mathcal{F}^{\prime}$.

Definition 4.4. A normal curve is a nontrivial (in the sense of Definition 4.2) connected component of a level set of $B$ such that $d B \neq 0$ at each of its points.

In this work, we use Sard's lemma for functions defined on $\mathbb{T} \times \mathbb{R}$, the statement of which we now recall.

Lemma 4.5 (Sard). If $f: \mathbb{T} \times \mathbb{R} \rightarrow \mathbb{R}$ is a smooth and surjective function, then the set of critical values of $f$ has Lebesgue measure zero. In particular, the set of regular values of $f$ is dense in $\mathbb{R}$. 
4.2. The reduction to the model case. We now proceed to describe the announced reduction. In order to achieve this we will rely on the notation and results of the above subsection.

We make the remark that we may work either with the case when $B$ has a disconnected sublevel, say, $\Omega_{\rho}$, or the case when $B$ has a disconnected superlevel, say, $\Omega^{\rho}$. For this, if necessary, we make a change of variables in $\mathbb{T}^{3}$, namely, $(t, x) \mapsto$ $(-t,-x)$. Note that the property (4.1) is preserved under this change of variables. In order to fix the ideas we will concentrate - for now - on the case of a disconnected sublevel.

Since every sublevel of $B$ contains a unique unbounded connected component (which contains an upper half-cylinder), the sublevel $\Omega_{\rho}$ has at least one bounded connected component which we denote by $\Omega_{\rho}^{b}$.

We may assume that $\rho$ is a regular value of $B$; indeed, if $\rho$ is a critical value, then Sard's lemma implies the existence of a regular value $r \in \mathbb{R}$, with $\rho>r$, such that $B\left(t^{*}\right)=r$ for some $t^{*} \in \Omega_{\rho}^{b}$. Since $B\left(t^{*}\right)<\rho$ and $B(t)=\rho$, for all $t \in \partial \Omega_{\rho}^{b}$, we have $\mathcal{F}^{\prime}\left(t^{*}\right) \subset \Omega_{\rho}^{b}$. Since $r$ is a regular value it follows from Proposition $4.3 \mathrm{k} v$, that $\mathcal{F}^{\prime}\left(t^{*}\right)$ has a neighborhood foliated by level curves, all of them with distinct values of $B$. Therefore $B$ has a regular value $r$, whose sublevel $\Omega_{r}$ is disconnected and has a bounded connected component, $\Omega_{r}^{b}$, such that $\mathcal{F}^{\prime}\left(t^{*}\right) \subset \partial \Omega_{r}^{b}$, which concludes the proof of our claim.

We claim that $\partial \Omega_{\rho}^{b}$ is equal to a finite union of connected components of $\mathcal{F}(t)$ for some $t \in \partial \Omega_{\rho}^{b}$. Indeed, $B$ is equal to $\rho$ on $\partial \Omega_{\rho}^{b}$ and $\rho$ is a regular value, hence it follows from Proposition $4.3 \mathrm{i} i \mathrm{i}$ that $\partial \Omega_{\rho}^{b}$ is contained in a finite union of connected components of $\mathcal{F}(t), t \in \partial \Omega_{\rho}^{b}$. On the other hand, if $t \in \partial \Omega_{\rho}^{b}$ then, by Proposition 4.3 ; , we obtain $\mathcal{F}^{\prime}(t) \subset \partial \Omega_{\rho}^{b}$, which concludes the proof of our claim.

We may write

$$
\partial \Omega_{\rho}^{\mathrm{b}}=\left(\bigcup_{i=1}^{N_{0}} \alpha_{i}\right) \cup\left(\bigcup_{j=1}^{N_{1}} \gamma_{j}\right), \quad N_{0}, N_{1} \geq 0, \quad N_{0}+N_{1} \geq 1,
$$

where each $\alpha_{i}$ is a trivial embedded copy of $\mathbb{T}$ and each $\gamma_{j}$ is a nontrivial embedded copy of $\mathbb{T}$ in the cylinder $\mathbb{T} \times \mathbb{R}$.

We claim that we have either $N_{1}=0$ or $N_{1}=2$.

It is easy to see that if we had $N_{1} \geqslant 3$, then $\Omega_{\rho}^{b}$ would be disconnected by one of the intermediate curves $\gamma_{j}$, which is a contradiction.

Assume now that $N_{1}=1$ and let $\gamma_{1}$ be the nontrivial curve in $\partial \Omega_{\rho}^{b}$; we may assume that $\gamma_{1}=\left\{t_{2}=c_{1}\right\}$. We will prove that a contradiction occurs.

First we consider the case when $\Omega_{\rho}^{b}$ lies below $\gamma_{1}$. Let $\bar{t}=\left(\bar{t}_{1}, \bar{t}_{2}\right) \in \Omega_{\rho}^{b}$, with $\bar{t}$ near $\gamma_{1}$. Let $V \doteq\left\{t_{2}<c_{1}\right\} \backslash \bigcup_{j=1}^{N_{0}} \Gamma_{j}$, where $\Gamma_{j}$ is the closed bounded region whose boundary is $\alpha_{j}$. Take $s=\left(s_{1}, s_{2}\right) \in V, s_{2}<\bar{t}_{2}$ such that $B(s)>\rho$. Since $V$ is path-connected, there exists a path $\beta$ entirely contained in $V$, connecting the points $\bar{t} \in \Omega_{\rho}^{b}$ and $s \in V \backslash \overline{\Omega_{\rho}^{b}}$, which implies that $\beta$ meets $\partial \Omega_{\rho}^{b}$, which is a contradiction.

Now we consider the case when $\Omega_{\rho}^{b}$ lies above $\gamma_{1}=\left\{t_{2}=c_{1}\right\}$. The proof is similar. Take $\bar{t} \in \Omega_{\rho}^{b}$ near $\gamma_{1}$, that is, $\bar{t}=\left(\overline{t_{1}}, \overline{t_{2}}\right), \bar{t}_{2}>c_{1}, \bar{t}_{2}$ close to $c_{1}$.

Let $V \doteq\left\{t_{2}>c_{1}\right\} \backslash \bigcup_{j=1}^{N_{0}} \Gamma_{j}$ be as before. We know that the unique unbounded connected component of the sublevel $\{B(t)<\rho\}$ contains an upper half-cylinder. Hence $V$ contains a point $s=\left(s_{1}, s_{2}\right)$ such that $s \notin \overline{\Omega_{\rho}^{b}}$. Since $\bar{t}, s \in V$ and $s \neq \bar{t}$, 
there exists a path $\beta$ entirely contained in $V$ connecting $\bar{t}$ and $s$. But then $\beta$ must cross $\partial \Omega_{\rho}^{b}$, a contradiction. Hence $N_{1} \neq 1$.

We have completed the proof of our claim, namely, that either $N_{1}=0$ or $N_{1}=2$.

In order to go on with the reduction to the model case, it is important to distinguish between two cases which we proceed to describe.

Case 1. There exists a connected component of a regular bounded sublevel (or superlevel) such that $N_{1}=2$, that is, its boundary has exactly two nontrivial connected components of the level set of $B$ in the cylinder.

Case 2. The boundary of every connected component of a bounded regular sublevel or superlevel of $B$ contains only trivial copies of the unit circle $\mathbb{T}$.

We begin by dealing with Case 1 .

We may assume that the nontrivial connected components of the level set $\rho$ are $\left\{t_{2}=c_{1}\right\}$ and $\left\{t_{2}=c_{2}\right\}$, where $c_{2}>c_{1}$.

Let $t_{0} \in \mathbb{T} \times\left[c_{1}, c_{2}\right]$ be a point of global minimum of $B$ on $\mathbb{T} \times\left[c_{1}, c_{2}\right]$; note that $t_{0}$ is a critical point of $B$, since the restriction of $B$ to $\overline{\Omega_{\rho}^{b}}$ attains a global minimum at a point of $\Omega_{\rho}^{b}$.

Let $\alpha:[0,2 \pi] \rightarrow \mathbb{T} \times \mathbb{R}$ be a smooth parametrization of $\left\{t_{2}=c_{2}\right\}$ such that $\alpha^{\prime}(s) \neq 0, s \in[0,2 \pi]$, and take $0<s_{1}<s_{2}<2 \pi$.

Since $\overline{\Omega_{\rho}^{b}}$ is a path-connected subset of the cylinder, we may replace $\alpha\left(\left[s_{1}, s_{2}\right]\right)$ by an arc, called $\gamma^{*}$, which contains the points $\alpha\left(s_{1}\right), t_{0}$ and $\alpha\left(s_{2}\right)$, and lies in $\overline{\Omega_{\rho}^{b}}$, in such a way that the curve $\sigma_{1} \doteq \alpha\left(\left[0, s_{1}\right]\right) \cup \gamma^{*} \cup \alpha\left(\left[s_{2}, 2 \pi\right]\right)$ is a nontrivial non-self-intersecting smooth curve in the cylinder.

We observe that for $t=\left(t_{1}, t_{2}\right) \in \mathbb{T} \times \mathbb{R} \backslash \Omega_{\rho}^{b}$ near $\alpha([0,2 \pi])$ with $t_{2}>c_{2}$ we have

$$
B(t)>\rho .
$$

We may assume that $\sigma_{1}$ is $\mathbb{T} \times\{0\}, t_{0}=(0,0)$ and $B(0,0)=0$.

It follows from (4.4) that the global maximum of $B$ on $\mathbb{T} \times[0,2 \pi]$ occurs in $t_{\max } \in \mathbb{T} \times(0,2 \pi)$ and we may consider another nontrivial smooth curve, $\sigma_{2}$, without self-intersection (for example, the graph of a function of $t_{2}$ ) which contains the points $(0,0), t_{\max }$ and $(0,2 \pi)$. We may assume that $\sigma_{2}$ is $\{0\} \times \mathbb{T}$, finishing the reduction to the model case in Case 1 .

It remains to treat Case 2. We begin with a preliminary result.

Proposition 4.6. Assume that

$\left({ }^{*}\right)$ : the boundary of every bounded regular sublevel or superlevel of $B$ contains only trivial copies of the unit circle $\mathbb{T}$.

Then

i. every regular level set contains exactly one nontrivial copy of $\mathbb{T}$;

ii. the nontrivial curve $\gamma$ in $(i)$ is part of the boundary of both the unique unbounded connected component of superlevel and the unique unbounded connected component of sublevel (with the same level $r$ ); the superlevel set lies below $\gamma$ and the sublevel set lies above $\gamma$;

iii. the boundary of each unbounded, regular sublevel or superlevel contains exactly one nontrivial curve $\gamma$;

iv. if a normal curve $\gamma$ lies below another normal curve $\gamma^{\prime}$, then the value of $B$ over $\gamma$ is strictly greater than the value of $B$ over $\gamma^{\prime}$; in particular, distinct normal curves have distinct values of $B$. 
Proof. Proposition 4.3i $v$ says that every regular level set contains at least one nontrivial copy of $\mathbb{T}$. Now if this copy were not unique, then the region bounded by two consecutive nontrivial copies of $\mathbb{T}$ would contain a connected component of either a sublevel or a superlevel, the boundary of which would contain both of these nontrivial copies of $\mathbb{T}$ violating our assumption $(*)$. This concludes the proof of $i$.

From Proposition 4.3 we know that $\gamma$ has a foliated neighborhood and that $\gamma$ is contained in the boundary of a sublevel as well as in the boundary of a superlevel. It follows from our assumption $(*)$ that $i i$ holds true.

Property $i i i$ is an immediate consequence of $i$ and $i$.

We now move on to the proof of $i v$. Let $\gamma_{r}$ and $\gamma_{r^{\prime}}$ be nontrivial connected components of level sets, with levels $r$ and $r^{\prime}$, respectively, such that $d B \neq 0$ at each point of the curves $\gamma_{r}$ and $\gamma_{r^{\prime}}$.

Then $\gamma_{r}$ is the nontrivial part of the boundary of the unbounded connected component of superlevel, denoted by $\Omega^{u, r}$, and $\gamma_{r}^{\prime}$ is the nontrivial part of the boundary of $\Omega^{u, r^{\prime}}$. We may assume that $\gamma_{r}=\left\{t_{2}=c\right\}$ and $\gamma_{r}^{\prime}=\left\{t_{2}=c^{\prime}\right\}$.

Then $c<c^{\prime}$ if and only if $\gamma_{r} \subset \Omega^{u, r^{\prime}}$ if and only if $\Omega^{u, r} \subset \Omega^{u, r^{\prime}}$ if and only if $\{B(t)>r\} \subseteq\left\{B(t)>r^{\prime}\right\}$ if and only if $r>r^{\prime}$. The proof is complete.

Proposition 4.7. Assume that

$\left({ }^{*}\right)$ : the boundary of every bounded regular sublevel or superlevel of $B$ contains only trivial copies of the unit circle $\mathbb{T}$.

Assume that $r$ is a regular value of $B$ and that the superlevel set $\Omega^{r}=\{t \in$ $\mathbb{T} \times \mathbb{R} ; B(t)>r\}$ is disconnected.

Then there is a diffeomorphism of the three-dimensional torus such that $\gamma \doteq\left\{t_{2}=0\right\}$ is a normal curve which is the nontrivial part of the boundary of the unique unbounded connected component of the superlevel $\Omega^{r}$, and, furthermore, there is a point $p \in \mathbb{T} \times(0,2 \pi)$ such that $B(p) \geq r$.

Proof. We may assume that $r=0$. We may also start from the situation where the boundary of the unbounded connected component, $\mathcal{U}$, of the superlevel $\Omega^{0} \doteq\{t \in \mathbb{T} \times \mathbb{R} ; B(t)>0\}$ is already the normal curve $\gamma \doteq\left\{t_{2}=0\right\}$. What we have to prove is that there is a point $p \in\left\{0<t_{2}<2 \pi\right\}$ such that $B(p) \geq 0$.

Take $V$ to be a bounded connected component of the superlevel $\Omega^{0}$. By assumption $(*)$ the boundary of $V$ consists of a finite number of trivial copies of $\mathbb{T}$. Since $\gamma$ is a normal curve, it has a neighborhood, $W$, foliated by nontrivial copies of $\mathbb{T}$. It follows that $V$ cannot intersect any translate of $W$ in the $t_{2}$ direction by numbers of the form $2 k \pi, k \in \mathbb{Z}$. We conclude that there is $k \in \mathbb{Z}$ such that $V \subset \bar{V} \subset\left\{2 k \pi<t_{2}<2(k+1) \pi\right\}$.

If $k=0$, then any point $p$ in the boundary of $V$ satisfies $B(p)=0$.

If $k>0$ and if $p=\left(p_{1}, p_{2}\right) \in \partial V$, then $p^{\prime} \doteq\left(p_{1}, p_{2}-2 k \pi\right) \in\left\{0<t_{2}<2 \pi\right\}$ and $B\left(p^{\prime}\right)=-2 k b_{20}>0$.

If $k<0$ we use the change of variables $(x, t) \rightarrow(-x,-t)$ and fall back into one of the previous two cases. The proof is complete.

Proposition 4.8. Assume that $\gamma \doteq\left\{t_{2}=0\right\}$ is a normal curve, $B$ is equal to 0 over $\gamma$ and $B$ has a critical point in $\mathbb{T} \times(0,2 \pi)$. Consider the set $W$ consisting of all $t \in \mathbb{T} \times(0,2 \pi)$ for which there is a normal curve $\alpha$ containing $t$ and such that the region bounded by $\gamma$ and $\alpha$ is foliated by normal curves. Then there is a point $t_{0} \in \partial W$ such that $d B\left(t_{0}\right)=0$ and $B\left(t_{0}\right)<0$. 
Proof. Clearly, $W$ is open, nonempty and connected. Also, $d B(t) \neq 0$, for all $t \in W$, hence $W$ is a proper subset of $\mathbb{T} \times(0,2 \pi)$. We may write the boundary of $W$ as a disjoint union $\partial W=\gamma \cup \mathcal{K}$; we call $\mathcal{K}$ the upper part of $\partial W$. Then $\mathcal{K}$ is a compact subset of a level set, with level $<0$ because $B$ is strictly decreasing as one moves transversally to the normal curves in $W$, away from the initial curve $\gamma$. If we had $d B(t) \neq 0$, for each $t \in \mathcal{K}$, then $K$ would be a normal curve contained in $W$, a contradiction which ends the proof.

Proposition 4.9. In the geometrical situation of the previous propositions, assume that $B$ satisfies $B\left(t_{0}\right)=0$ and $B(p)>0$.

Define $M$ by $M \doteq \max _{t \in \mathbb{T} \times[0,2 \pi]} B(t)$; then $M \geq B(p)>0$. Let $0<\delta<1$ be such that $B(t)<M / 2$, for all $t$ in the open disc $D\left(t_{0} ; \delta\right)$. Then there exists a nontrivial smooth simple curve $\sigma_{1}: \mathbb{T} \rightarrow \mathbb{T} \times \mathbb{R}$ with $\sigma_{1}(0)=t_{0}$ satisfying

i. $B\left(\sigma_{1}(\tau)\right)<M / 2, \tau \in \mathbb{T}$;

ii. the global maximum $M$ of $B$ on $\mathbb{T} \times[0,2 \pi]$ will be attained in the upper half-cylinder relatively to $\sigma_{1}$.

Proof. Let $\mathcal{F}^{\prime}(s)$ be a normal curve such that $\mathcal{F}^{\prime}(s) \cap D\left(t_{0}, \delta\right) \neq \varnothing$, which can be smoothly parametrized by $\gamma:[0,2 \pi] \rightarrow \mathbb{T} \times \mathbb{R}$ such that $\gamma^{(k)}(0)=\gamma^{(k)}(2 \pi)$, $k=0,1, \ldots$

By reparametrizing $\gamma$, if necessary, there exist $\tau_{1}<\tau_{2}$ such that $\gamma\left(\tau_{1}\right), \gamma\left(\tau_{2}\right)$ are in $\partial D\left(t_{0}, \delta\right)$ and $\gamma(\tau) \notin D\left(t_{0}, \delta\right)$ if either $\tau<\tau_{1}$ or $\tau>\tau_{2}$.

We now replace the arc $\gamma\left(\left(\tau_{1}, \tau_{2}\right)\right)$ by the juxtaposition of the segments $\left[\gamma\left(\tau_{1}\right), t_{0}\right]$ and $\left[t_{0}, \gamma\left(\tau_{2}\right)\right]$ obtaining a nontrivial, continuous, piecewise smooth curve $\tilde{\gamma}$ passing through $t_{0}$. Furthermore, $B(\tilde{\gamma}(\tau))<\frac{M}{2}, \tau \in \mathbb{R}$.

Finally, we smooth out $\tilde{\gamma}$ to obtain a curve $\sigma_{1}$ as required.

We are now finally ready to finish the reduction to the model case. We observe that $\max _{t \in \mathbb{T} \times[0,2 \pi]} B(t)=B\left(t_{\max }\right)$, where $t_{\max } \in \mathbb{T} \times(0,2 \pi)$, in particular, the maximum does not occur in $\mathbb{T} \times\{0\}$.

We take a nontrivial smooth simple curve, $\sigma_{2}$ (for example, the graph of a function of $\left.t_{2}\right)$ passing through the points $t_{0}, t_{\max }$ and $t_{0}+(0,2 \pi)$.

By making use of a smooth change of variables we may take $\sigma_{1}$ to be $t_{2}=0$ and $\sigma_{2}$ to be $t_{1}=0$. This finishes the reduction to the model case in the remaining case 2 .

\section{SuFFICIENCY}

In this section we prove that $\mathbb{L}^{0}$ is globally solvable on $\mathbb{T}^{3}$ if all sublevels $\Omega_{r}$ and all superlevels $\Omega^{r}$ of $B$ are connected.

Using Remark 2.5 and the arguments in the beginning of the previous section, it is enough to consider the case when $b_{10}=0$ and $b_{20}<0$.

Suppose, for the sake of reasoning, that there is a solution $u \in \mathcal{D}^{\prime}\left(\mathbb{T}^{3}\right)$ to $\mathbb{L}^{0} u=f$ for some $f$ in the space $\mathbb{E}$ of Definition 2.3. Then, the $x$-Fourier coefficients of $u$ must satisfy the equation

$$
\left(d_{t}-n b(t) \wedge\right) \hat{u}(t, n)=\hat{f}(t, n),(t, n) \in \mathbb{T}^{2} \times \mathbb{Z} .
$$

For each $n \in \mathbb{Z}$, we lift this equation to $\mathbb{T} \times \mathbb{R}$ and proceed to analyze it.

Let $t_{0} \in \mathbb{T} \times[0,2 \pi]$. 
For each $n \in \mathbb{Z}$ the general solution defined on $\mathbb{T} \times \mathbb{R}$ is given by

$$
\hat{u}(t, n)=\int_{t_{0}}^{t} \omega(t, n)+K_{n} e^{n B(t)},
$$

where $\omega(t, n)$ is the exact 1 -form

$$
\omega(t, n)(\tau) \doteq e^{-n[B(\tau)-B(t)]} \hat{f}(\tau, n), \quad \tau \in \mathbb{T} \times \mathbb{R},
$$

and $K_{n}$ is an arbitrary constant.

If $n=0$, then

$$
\hat{u}(t, 0)=\int_{t_{0}}^{t} \hat{f}(t, 0)+K_{0} .
$$

In fact, for $n \neq 0, K_{n}$ must be chosen so that $\hat{u}(\cdot, n)$ is a $2 \pi$-periodic function in the variable $t_{2}$; this yields a unique solution which may be written as

$$
\hat{u}\left(\gamma_{ \pm}(s), n\right)=\int_{0}^{s} g(\sigma, n) d \sigma+\frac{1}{e^{\mp n b_{20}}-1} \int_{0}^{2 \pi} g(\sigma, n) d \sigma,
$$

where $g$ is the function

$$
g(\sigma, n)=e^{-n\left[B\left(\gamma_{ \pm}(\sigma)\right)-B\left(\gamma_{ \pm}(s)\right)\right]} \hat{f}\left(\gamma_{ \pm}(\sigma), n\right) \cdot \gamma_{ \pm}{ }^{\prime}(\sigma), \quad \sigma \in[0,2 \pi],
$$

and $\gamma_{ \pm}:[0,2 \pi] \rightarrow \mathbb{T} \times \mathbb{R}$ are paths starting at a point $t_{0}$ and ending at $t_{0}+(0, \pm 2 \pi)$, respectively, whose projections on $\mathbb{T}^{2}$ are nontrivial smooth closed curves.

We will show that there are integration paths by means of which adequate estimates for the behavior of these solutions can be proved, and from this we will be able to obtain a true solution to the equation $\mathbb{L}^{0} u=f$.

5.1. Integration paths. The next results and proofs will show how the integration paths are chosen; they are based on propositions 2.3, 2.4 and 2.9 from [6].

Proposition 5.1. In the commensurable case, assume that all sublevels and all superlevels are connected. Then there is $M>0$ such that, for all $t_{0} \in \mathbb{T} \times[0,2 \pi]$ and $n \in \mathbb{Z}$, there exist smooth paths $\gamma_{ \pm}=\gamma_{ \pm}\left(t_{0}, n\right)$ joining the points $t_{0}$ and $t_{0}+(0, \pm 2 \pi)$ with length $\left|\gamma_{ \pm}\right|$satisfying

$$
\left|\gamma_{ \pm}\right|<M(1+|n|), \forall n \in \mathbb{Z}
$$

and such that

$$
\begin{aligned}
& \text { i. } B(\tau) \leqslant B(t)+\frac{1}{1+|n|}, \text { for all } \tau \in \gamma_{+} ; \\
& \text {ii. } B(\tau) \geqslant B(t)-\frac{1}{1+|n|}, \text { for all } \tau \in \gamma_{-} .
\end{aligned}
$$

Proof. We will provide details only for the construction of the path $\gamma_{+}$; similarly, one can prove the results concerning $\gamma_{-}$.

Let $\alpha=\min _{t \in \mathbb{T} \times[0,2 \pi]} B(t)$ and $\beta=1+\max _{t \in \mathbb{T} \times[0,2 \pi]} B(t)$ and consider the compact interval $J \doteq[\alpha, \beta]$. Then there exists $\nu>0$ such that $\mathbb{T} \times(\nu, \infty) \subset \Omega_{r}$ and $\mathbb{T} \times(-\infty,-\nu) \subset \Omega^{r}$ for all $r \in J$.

Set $K_{r} \doteq \Omega_{r} \cap(\mathbb{T} \times[-\nu, \nu])$. It is not difficult to prove that $K_{r}$ is bounded and path-connected.

For each $j \in \mathbb{N}$, consider the collection of all squares contained in $[0,2 \pi] \times \mathbb{R}$ having side length $2 \pi / 2^{j}$ and vertices in $\left(2 \pi / 2^{j}\right) \mathbb{Z}^{2}$.

Since $\mathbb{T} \times \mathbb{R}$ is locally isometric to $[0,2 \pi] \times \mathbb{R}$ the collection above induces a collection $\mathcal{D}_{j}$ of sets, which we will continue to call squares, in $\mathbb{T} \times \mathbb{R}$. 
For each $t \in \mathbb{T} \times[0,2 \pi]$ and $n \in \mathbb{Z}$, we have

$$
r \doteq B(t)+\frac{1}{2(1+|n|)} \in J
$$

Consider the sets $\mathcal{A}_{j r}=\left\{Q \in \mathcal{D}_{j} ; Q \cap K_{r} \neq \varnothing\right\}$ and $\mathcal{V}_{j r}=\bigcup_{Q \in \mathcal{A}_{j r}} Q$.

Observe that any point $s \in \mathcal{V}_{j r}$ is contained in a square $Q \subset \mathbb{T} \times \mathbb{R}$ with diagonal equal to $\pi \sqrt{2} / 2^{j-1}$, which intersects $K_{r}$ at a point $s^{\prime}$, hence

$$
B(s) \leqslant\left|B(s)-B\left(s^{\prime}\right)\right|+B\left(s^{\prime}\right) \leqslant\|b\|_{\infty}\left|s-s^{\prime}\right|+r \leqslant\|b\|_{\infty} \frac{\pi \sqrt{2}}{2^{j-1}}+r .
$$

Now we take $j \in \mathbb{N}$ such that $2^{j-1}<4 \pi \sqrt{2}(1+|n|)|| b \|_{\infty} \leqslant 2^{j}$, and we have

$$
B(s) \leqslant\|b\|_{\infty} \frac{\pi \sqrt{2}}{2^{j-1}}+r \leqslant \frac{1}{2(1+|n|)}+r=B(t)+\frac{1}{1+|n|}, \forall s \in \mathcal{V}_{j r} .
$$

$K_{r}$ is path-connected, hence so is $\mathcal{V}_{j r}$, thus we can join the points $t$ and $t+(0,2 \pi)$ by means of a path $\gamma_{+}$which is piecewise linear (in the sense that it is made up of sides and diagonals of the squares above), is contained in $\mathcal{V}_{j r}$, and intersects each square in $\mathcal{V}_{j r}$ at most once.

If $N$ is the number of squares in $\mathcal{D}_{0}$ intersecting $K_{r}$, then the number of squares in $\mathcal{D}_{j}$ intersecting $K_{r}$ is at most $4^{j} N$. Since the diagonal of each square in $\mathcal{V}_{j r}$ is $\pi \sqrt{2} / 2^{j-1}$, we have

$$
\left|\gamma_{+}\right| \leqslant \frac{\pi \sqrt{2}}{2^{j-1}} 4^{j} N<(4 \pi \sqrt{2})^{2} N|| b||_{\infty}(1+|n|)=M(1+|n|) .
$$

It is standard that $\gamma_{+}$can be replaced by a smooth path satisfying similar estimates.

5.2. Construction of the solution. For each $f \in \mathbb{E}$, we will show that the equation $\mathbb{L}^{0} u=f$ has a solution. In fact, we prove that the formal partial $x$-Fourier series $u(t, x)=\sum \hat{u}(t, n) e^{i n x}$, with coefficients given by (5.3) and (5.4), is a $C^{\infty}$ solution to this equation.

Lemma 5.2. For each pair $(\alpha, \beta)$ of nonnegative integers, with $\alpha+\beta \geqslant 1$ and for each $n \neq 0$, we have

$$
\partial^{(\alpha, \beta)}\left(\int_{t_{0}}^{t} \omega(t, n)\right)=n^{\alpha+\beta} F_{(\alpha, \beta)}\left(\int_{t_{0}}^{t} \omega(t, n)\right)+n^{\alpha+\beta-1} G_{(\alpha, \beta)},
$$

where

$$
F_{(p, q)+e_{j}}=\left\{\begin{array}{ccc}
b_{j} & \text { if } & p+q=0, \\
b_{j} F_{(p, q)}+\frac{1}{n} \partial_{j} F_{(p, q)} & \text { if } & p+q \geqslant 1
\end{array}\right.
$$

and

$$
G_{(p, q)+e_{j}}=\left\{\begin{array}{cl}
\hat{f}_{j} & \text { if } p+q=0, \\
\hat{f}_{j} F_{(p, q)}+\frac{1}{n} \partial_{j} G_{(p, q)} & \text { if } p+q \geqslant 1 .
\end{array}\right.
$$

Proof. Using the formula

$$
\partial_{j}\left(\int_{t_{0}}^{t} \omega(t, n)\right)=n b_{j}(t)\left(\int_{t_{0}}^{t} \omega(t, n)\right)+\hat{f}_{j}(t, n)
$$

and proceeding by induction on the order of differentiation, the lemma follows. 
Observe that $F_{(\alpha, \beta)}$ is bounded over $\mathbb{T} \times \mathbb{R}$ because $F_{(\alpha, \beta)}$ depends only on the derivatives of $b_{j}$ (of order less than $\alpha+\beta$ ) and on nonpositive powers of $n$. Moreover, $G_{(\alpha, \beta)}$ is a finite sum, each summand being uniformly bounded, and depending on the derivatives of $b_{j}$ and $\hat{f}_{j}$ (of order less than $\alpha+\beta$ ) and on nonpositive powers of $n$. Thus, given $(\alpha, \beta) \in \mathbb{Z}_{+}^{2}$ and $N>0$, we obtain a constant $C_{(\alpha, \beta)}$ such that $\left|G_{(\alpha, \beta)}\right| \leqslant C_{(\alpha, \beta)}(1+|n|)^{-N}$.

Lemma 5.3. Let $t_{0} \in \mathbb{T} \times[0,2 \pi]$. For every $N \in \mathbb{N}$, there exists a constant $C>0$ such that

$$
\left|\int_{t_{0}}^{t} \omega(t, n)\right| \leqslant \frac{C}{(1+|n|)^{N}}, \forall n \in \mathbb{Z},
$$

where $\omega(t, n)$ was defined in (5.2) and $t \in \gamma_{ \pm}$, where $\gamma_{ \pm}$is one of the paths obtained in Proposition 5.1 (this choice depends only on the sign of $n$ ).

Proof. For $n<0$, by Proposition 5.1 w we have $e^{-n(B(\tau)-B(t))} \leqslant e^{-n \frac{1}{1+|n|}} \leqslant e$, hence

$$
\left|\int_{t_{0}}^{t} \omega(t, n)\right| \leqslant \sup _{\tau \in \gamma_{+}} \mid e^{-n \frac{1}{1+|n|} \hat{f}(\tau, n) \mid C(1+|n|) \leqslant C(1+|n|)^{-N} .}
$$

If $n>0$, we use Proposition 5.1 $i i$ and also obtain (5.6). The case $n=0$ is trivial.

Definition 5.4. The operator $\mathbb{L}^{0}$ is globally solvable in the $C^{\infty}$ sense if, for each $f \in \mathbb{E}$, there exists a solution $u \in C^{\infty}\left(\mathbb{T}^{3}\right)$ to the equation $\mathbb{L}^{0} u=f$.

Proposition 5.5. Suppose that, for each $r \in \mathbb{R}$, the sublevel $\Omega_{r}$ and the superlevel $\Omega^{r}$ are connected. Then the operator $\mathbb{L}^{0}=d_{t}+i b(t) \wedge \partial_{x}$ is globally solvable in the $C^{\infty}$ sense.

Proof. Since $b_{20}<0$, there is a constant $C_{0}>0$ such that

$$
C_{0}^{-1} \leqslant\left|e^{\mp n b_{20}}-1\right| \leqslant C_{0}, \forall n \in \mathbb{Z}, \text { with } \pm n>0 .
$$

Lemma 5.3 implies $\left|\hat{u}\left(\gamma_{ \pm}(s), 0\right)\right| \leqslant C$ and

$$
\begin{aligned}
|\hat{u}(t, n)| & \leqslant\left|\int_{t_{0}}^{t} \omega(t, n)\right|+\frac{1}{\left|e^{\mp n b_{20}}-1\right|}\left|\int_{t_{0}}^{t_{0} \pm(0,2 \pi)} \omega(t, n)\right| \\
& \leqslant \frac{C^{\prime}}{(1+|n|)^{N}}, \text { for } \pm n>0 \text {, respectively. }
\end{aligned}
$$

When $\alpha+\beta \geqslant 1$, Lemma 5.2 implies

$$
\left|\partial^{(\alpha, \beta)} \hat{u}(t, n)\right| \leqslant|n|^{\alpha+\beta} \frac{C_{(\alpha, \beta), N}}{(1+|n|)^{N+\alpha+\beta}} C_{(\alpha, \beta)}^{\prime}+|n|^{\alpha+\beta-1} \frac{C_{(\alpha, \beta), N}^{\prime \prime}}{(1+|n|)^{N+\alpha+\beta-1}},
$$

thus, for each $N>0$, there is a constant $C>0$ such that,

$$
\left|\partial^{(\alpha, \beta)} \hat{u}(t, n)\right| \leqslant \frac{C}{(1+|n|)^{N}}, \forall n \neq 0, t \in[0,2 \pi]^{2},
$$

which implies that $u \in C^{\infty}\left(\mathbb{T}^{3}\right)$.

The proof of sufficiency in Theorem 2.4 is complete. 


\section{REFERENCES}

[1] A. Bergamasco, Remarks about global analytic hypoellipticity, Trans. Amer. Math. Soc. 351 (1999), 4113-4126. MR1603878 (99m:35032)

[2] A. Bergamasco, P. Cordaro, P. Malagutti, Globally hypoelliptic systems of vector fields, J. Funct. Anal. 114 (1993), 267-285. MR:1223704 (94e:35048)

[3] A. Bergamasco, P. Cordaro G. Petronilho, Global solvability for certain classes of undedetermined of vector fields, Math. Z. 223 (1996), 223-274. MR.1417431 (98e:58180)

[4] A. Bergamasco, P. Dattori da Silva, Global solvability for a special class of vector fields on the torus, in: Contemp. Math. vol. 400, Amer. Math. Soc., Providence, RI, (2006), 11-20. MR:2222462 (2006m:35024)

[5] A. Bergamasco, P. Dattori da Silva, Solvability in the large for a class of vector fields on the torus, J. Math. Pures Appl. 86 (2006), 427-447. MR2271625(2007i:35012)

[6] A. Bergamasco, A. Kirilov, Global solvability for a class of overdetermined systems, J. Funct. Anal. 252 (2007), 603-629. MR2360930 (2008k:35338)

[7] A. Bergamasco, A. Meziani, Solvability near the characteristic set for a class of planar vector fields of infinite type, Ann. Inst. Fourier (Grenoble) 55 (2005), 77-112. MR2141289 (2005m:35028)

[8] A. Bergamasco, W. Nunes, S. Zani, Global analytic hypoellipticity and pseudoperiodic functions, Math. Contemp. 18 (2000), 43-57. MR.1812862 (2001m:35062)

[9] A. Bergamasco, W. Nunes, S. Zani, Global properties of a class of overdetermined systems, J. Funct. Anal. 200 (2003), 31-64. MR1974087 (2004c:35295)

[10] A. Bergamasco, S. Zani, Prescribing analytic singularities for solutions of a class of vector fields on the torus, Trans. Amer. Math. Soc. 357 (2005), 4159-4174. MR.2159704 (2006c:35002)

[11] A. Bergamasco, S. Zani, Globally analytic hypoelliptic vector fields on compact surfaces, Proc. Amer. Math. Soc. 136 (2008), 1305-1310. MR2367104 (2009e:35031)

[12] A. Bergamasco, S. Zani, Global analytic regularity for structures of co-rank one, Comm. Partial Differential Equations 33 (2008), 933-941. MR2424383 (2009i:35048)

[13] S. Berhanu, P. Cordaro, J. Hounie An Introduction to Involutive Structures. New Mathematical Monographs, 6. Cambridge University Press, Cambridge, 2008. MR2397326|(2009b:32048)

[14] F. Cardoso, J. Hounie, Global solvability of an abstract complex, Proc. Amer. Math. Soc. 65 (1977), 117-124. MR0463721 (57:3663)

[15] J. Hounie, Globally hypoelliptic and globally solvable first order evolution equations, Trans. Amer. Math. Soc. 252 (1979), 233-248. MR534120 (80h:35035)

[16] A. Meziani, Hypoellipticity of nonsingular closed 1-forms on compact manifolds, Comm. Partial Differential Equations 27 (2002), 1255-1269. MR1924466 (2003f:58002)

[17] F. Treves, Study of a model in the theory of complexes of pseudodifferential operators, Ann. Math. (2) 104 (1976), 269-324. MR0426068 (54:14014)

[18] F. Treves, Hypoanalitic Structures (Local Theory), Princeton University Press, Princeton, NJ, 1992. MR.1200459 (94e:35014)

Departamento de Matemática, iCMC-UsP, Caixa Postal 668, 13560-970, São Carlos, SP. BRAZIL

E-mail address: apbergam@icmc.usp.br

Departamento de Matemática, UfPr, Caixa Postal 19081, Curitiba, PR, 81531-990, BRAZIL

E-mail address: akirilov@ufpr.br

Departamento de Matemática, ICMC-UsP, Caixa Postal 668, 13560-970, São Carlos, SP, BRAZIL

E-mail address: wvlnunes@icmc.usp.br

Departamento de Matemática, iCMC-Usp, Caixa Postal 668, 13560-970, São Carlos, SP, BRAZIL

E-mail address: szani@icmc.usp.br 